\title{
ERRATA
}

\section{Continuous directed evolution of aminoacyl-tRNA synthetases}

David I Bryson, Chenguang Fan, Li-Tao Guo, Corwin Miller, Dieter Söll \& David R Liu

Nat. Chem. Biol. 13, 1253-1260 (2017); published online 16 October 2017; corrected after print 1 December 2017

In the version of this article initially published, the label colors for $p$-NF and $p$-IF in the key for Figure $4 \mathrm{c}$ were transposed. The cyan bars should correspond to $p$-NF and the fuchsia bars to $p$-IF. The error has been corrected in the HTML and PDF versions of the article. 